\title{
Scaling up perioperative medicine for older people undergoing surgery (POPS) services; use of a logic model approach
}

\author{
Authors: Emily V Jasper, ${ }^{A}$ Jugdeep K Dhesi, ${ }^{B}$ Judith SL Partridge ${ }^{C}$ and Nick Sevdalis ${ }^{D}$
}

\begin{abstract}
Perioperative medicine for older people undergoing surgery (POPS) services are gaining traction, in acknowledgment of the poorer outcomes experienced by older surgical patients. In response to the NHS' growing focus on scaling innovation, a logic model of the POPS service at Guy's and St Thomas' NHS Foundation Trust was developed to articulate a founding centre's experience. The logic model was applied as a means of service evaluation and to guide implementation of a new POPS service at a district general trust. This is a novel study within the field of perioperative medicine for older people, interlinking implementation science theory to achieve meaningful clinical results and describe the lessons learnt during the process. Future work will include validation of this logic model to facilitate national POPS scale-up.
\end{abstract}

KEYWORDS: Perioperative medicine, older people, geriatric surgical liaison, logic model, implementation science

\section{Introduction}

The surgical population in England is ageing at a faster rate than the general population. ${ }^{1}$ In addition to surgical pathology, older patients present with age-related physiological deterioration, multimorbidity and geriatric syndromes including frailty. These factors are associated with poorer postoperative outcomes and

Authors: ${ }^{A}$ research registrar, perioperative medicine for older people undergoing surgery (POPS) team, Guy's and St Thomas' NHS Foundation Trust, London, UK; ${ }^{B}$ consultant geriatrician, perioperative medicine for older people undergoing surgery (POPS) team Guy's and St Thomas' NHS Foundation Trust, London, UK, honorary reader, Faculty of Life Sciences and Medicine, King's College London, London, UK and honorary associate professor, Division of Surgery \& Interventional Science, University College London, London, UK; ${ }^{C}$ consultant geriatrician, perioperative medicine for older people undergoing surgery (POPS) team, Guy's and St Thomas' NHS Foundation Trust, London, UK and honorary senior lecturer, Faculty of Life Sciences and Medicine, King's College London, London, UK; D professor of implementation science and patient safety, Centre for Implementation Science, King's College London, London, UK increased financial cost. ${ }^{1}$ National projections forecast a linear increase in numbers of older surgical patients, in part related to demographic change and surgical advances, that presents ongoing challenges to the delivery of quality healthcare for this population. To address these challenges, novel models of perioperative care have been developed across the UK and internationally. ${ }^{2,3}$ One of the best established and evidenced programmes is the perioperative medicine for older people undergoing surgery (POPS) service.

POPS was established in 2003 at Guy's and St Thomas' NHS Foundation Trust (GSTFT) as one of the first services to respond to the needs of the complex older surgical population. POPS is a geriatrician-led multidisciplinary service that uses comprehensive geriatric assessment (CGA) methodology throughout the surgical pathway. Preoperatively, elective patients are assessed and medically and functionally optimised prior to shared decision making undertaken collaboratively with surgical and anaesthetic colleagues. Postoperatively, inpatient care uses a shared-care model between geriatricians and surgical teams for both elective and emergency surgery. On the surgical wards, the POPS team provides medical input, directs rehabilitation and facilitates safe, effective, timely discharge from hospital.

Following more than a decade of development, POPS is now partnered with all surgical specialties and the anaesthetic directorate at GSTFT with joint ward rounds, board rounds and multidisciplinary team meetings. The benefits of such CGA-based services have been demonstrated across a number of specialties, including orthopaedics, urology, vascular and general surgery, with reductions observed in length of stay, postoperative morbidity and mortality for elective and emergency surgical patients. ${ }^{4-7}$ The UK's royal colleges of surgeons, anaesthetists and physicians advocate such collaborative services attuned to the needs of complex older surgical patients. ${ }^{8-10}$

However, despite the evidence and national endorsement, a UK's survey in 2019 identified that almost half of all acute NHS trusts do not yet provide any such services. ${ }^{2}$ This inequality across the UK highlights the need for systematic scale-up of a service such as POPS. The challenges in such a scale-up are not unique to POPS. The implementation gap is well recognised in the literature with a delay of 17 years cited between evidence being available and widespread clinical services being established. ${ }^{11}$ Further to translational delays, scaling up and disseminating innovative models of care across a healthcare system also requires fidelity to 
the original intervention while ensuring sensitive adaptation to the local context. To meet these challenges, broad stakeholder buy-in, organisational readiness and efficient use of scarce resources (funding and workforce) are necessary. ${ }^{12-14}$ Ensuring fidelity refers to the delivery of interventions as designed and intended and applies particularly to complex multicomponent interventions such as the POPS service. Addressing these translational challenges is necessary to establish effective, efficient and equitable services for older surgical patients across the UK.

One approach to the effective scale-up of a complex intervention, while maintaining fidelity, is through development of a logic model. ${ }^{15,16} \mathrm{~A}$ logic model is a graphic designed to demonstrate the theory of how an intervention achieves short- and long-term impact and provides detail on the core components, the mechanism and the context necessary to effect the intended change. Stakeholder input into the logic model design is critical to ensure fidelity and provide sufficient detail to ensure clinical utility. ${ }^{15,16}$ Through a visual framework (usually on a single page or diagram), logic models provide stakeholders with a figurative road map to facilitate daily practice and assist with planning and instituting scale-up activities.

This study outlies the development of a logic model to describe enablers and address barriers to the widespread scale-up of POPS services within the NHS. In addition, the paper evaluates feasibility and utility of the logic model in facilitating translation of the POPS service from GSTFT (POPS@GSTFT) to Dartford and Gravesham NHS Trust (DGT), a district general hospital (POPS@DGT).

\section{Methods: developing the model}

The process of developing a logic model for the POPS@GSTFT involved recruiting a panel of 13 experts, including eight clinical experts, three service managers within the service and two implementation science and patient safety experts. The clinical experts represented perioperative geriatricians, anaesthetists, surgeons and clinical nurse specialists who were working members of the POPS team at GSTFT (the host site) or DGT (the translation site). The panel members and affiliations are listed in supplementary material S1.

The logic model was developed using a four-stage process; familiarisation with the logic model concept, literature review, logic model drafting and expert panel review. The implementation science experts were fundamental in stage 1 , through acquainting the panel with the structure and role of a logic model. In stages $2-4$, three panellists (two perioperative geriatricians and one implementation science and safety expert) were responsible for drafting the logic model, guided by their experience of establishing a POPS service, a literature review and implementation science expertise. The draft model was built through mapping themes and ideas against the core components identified in the WK Kellogg Foundation Logic model development guide. ${ }^{15}$

The panellists met for three sessions (facilitated by the implementation scientists) within a 3-month period, with each session focused on a staged review of the draft model components. The panellists' input at each meeting was essential in amending the model and ensuring the model contents were accurate and user-friendly. If a panellist was unable to attend a meeting, the resources and discussion were distributed and absent panellists contributed their thoughts electronically. Between meetings, panellists were sent the updated version of the logic model and the previous minutes. Final amendments were made to the model after it was used to guide implementation at DGT, to ensure the model reflected insights learnt during that period. The study did not require approval from the local research ethics committee.

\section{Results}

The final logic model produced through this multistage process is shown in Table 1. In the sections that follow, the lessons learnt and outcomes from the process of logic model development are discussed.

\section{Developing the logic model: style}

A key decision in the model construction process was the choice of logic model style, with the literature reporting theory, activity or outcome focused model styles. ${ }^{15}$ An activity-based model pays close attention to implementation specifics and constructs a detailed map for future users. ${ }^{15}$ The panellists selected this option for the POPS logic model in conjunction with a 'drilled down' approach, allowing the inclusion of high level detail within each model element. ${ }^{15}$ This has the two-fold benefit of engaging current stakeholders in the creation of a model, through reliance on their expertise to accurately document the process, but second, creating a document that is specific and practical for future scale-up. Technically, such logic models offer implementation blueprints, which are a key strategy for the successful scale up of an evidenced programme, such as POPS. An example of this detail was evident in the approach to documenting POPS service long-term outcomes where the panel provided explicit measures to avoid the recognised limitation of vague or non-specific outcome measures. ${ }^{15}$ These explicit measures were further categorised into patient, service and implementation outcomes to maintain clarity during the translation process, while ensuring the model was feasible with face validity to future stakeholders.

\section{Developing the logic model: stakeholder input}

Implementation science literature consistently emphasises the importance of wide stakeholder input in logic model design. This informed the selection of a broad expert panel representative of all stakeholders in the perioperative pathway. The value of the diverse panel became apparent during the logic model development process; the variety of stakeholder experiences and expertise ensured a level of detail and direction that could not have been achieved with a less inclusive panel. Patient and carer input was actively sought, through the patient and public involvement (PPI) group at DGT. While the patient group did not attend the logic model meetings, their input was used to refine the final logic model.

\section{Developing the logic model: novel components}

The panel agreed that the logic model needed to outline the overarching values of the POPS service, while accurately describing the daily processes undertaken by the clinical team (both core values and operational elements). This approach allowed the panel to define a set of 'core components' which are recognised in the literature as the key 'active ingredients' of a complex intervention, those that must be delivered as part of scale up if the intervention is to achieve clinical effectiveness. ${ }^{13,17}$ For example, the addition of one core component, 'facilitate proactive liaison 


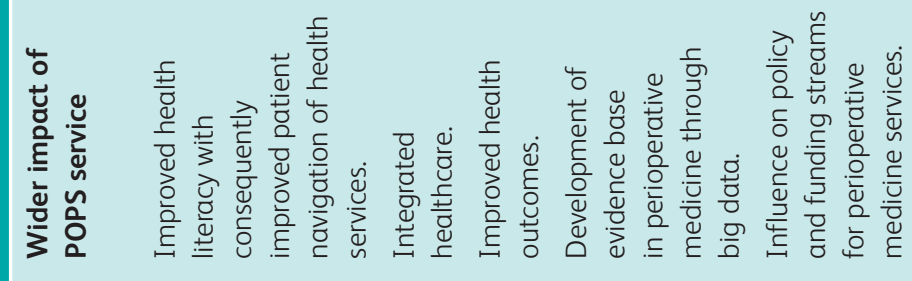

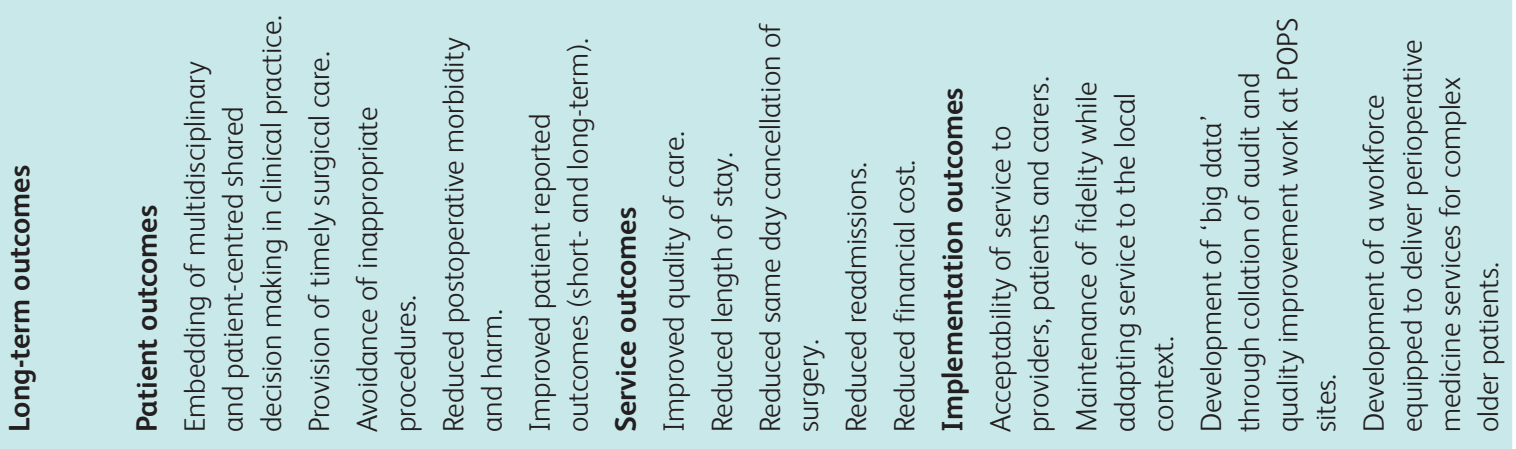

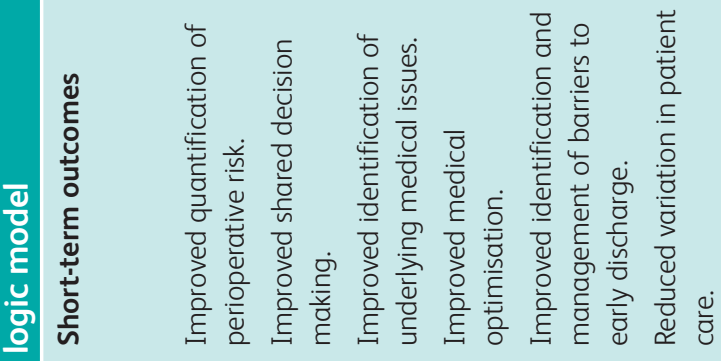

กิ

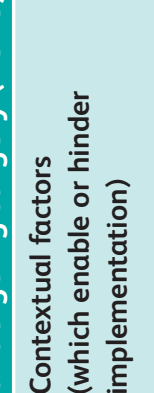

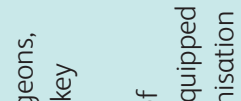

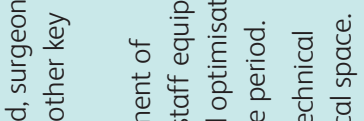

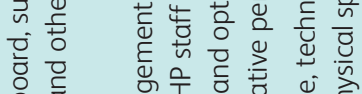

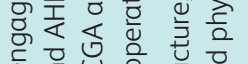

E⿱

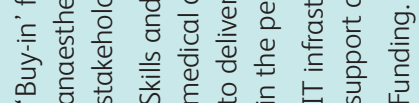

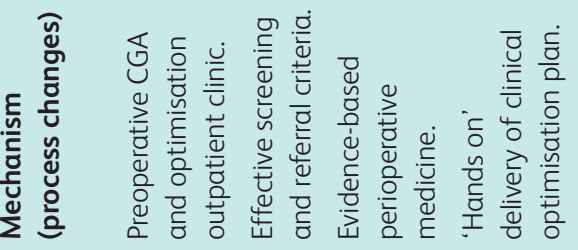

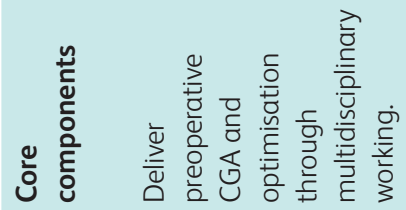

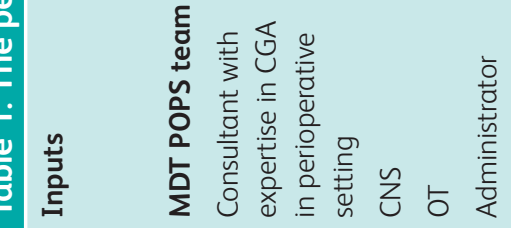




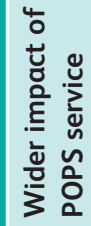

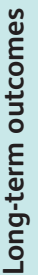

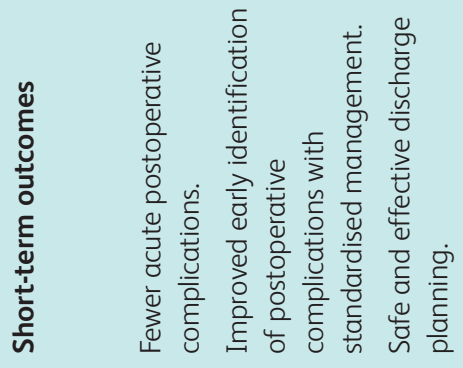

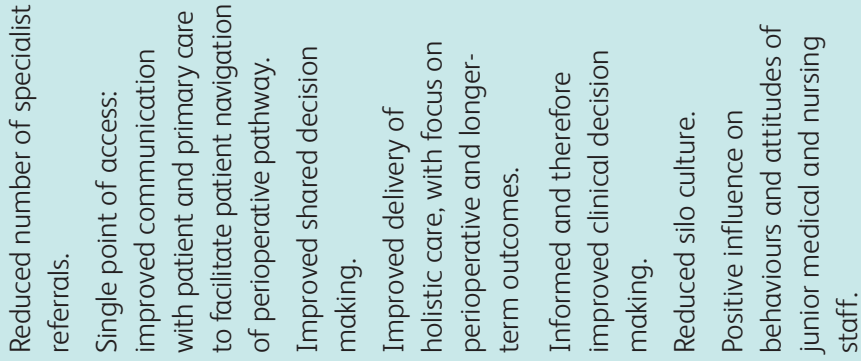
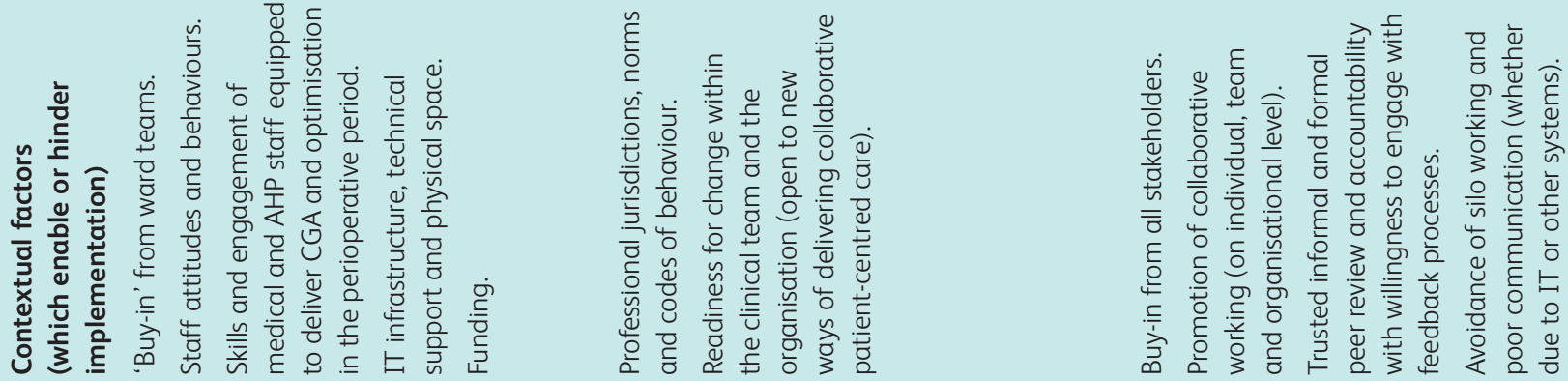

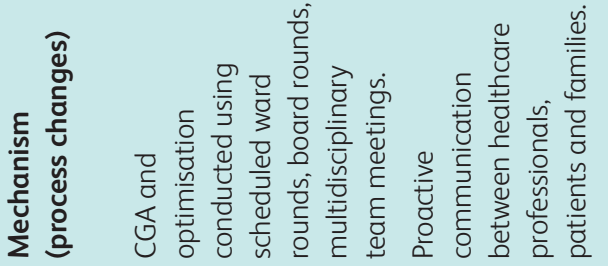
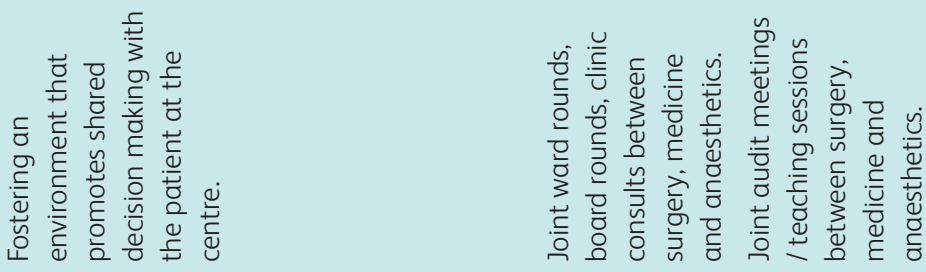

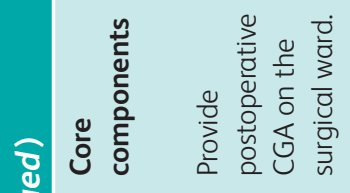

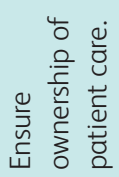

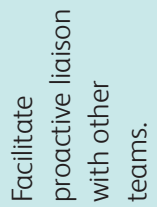




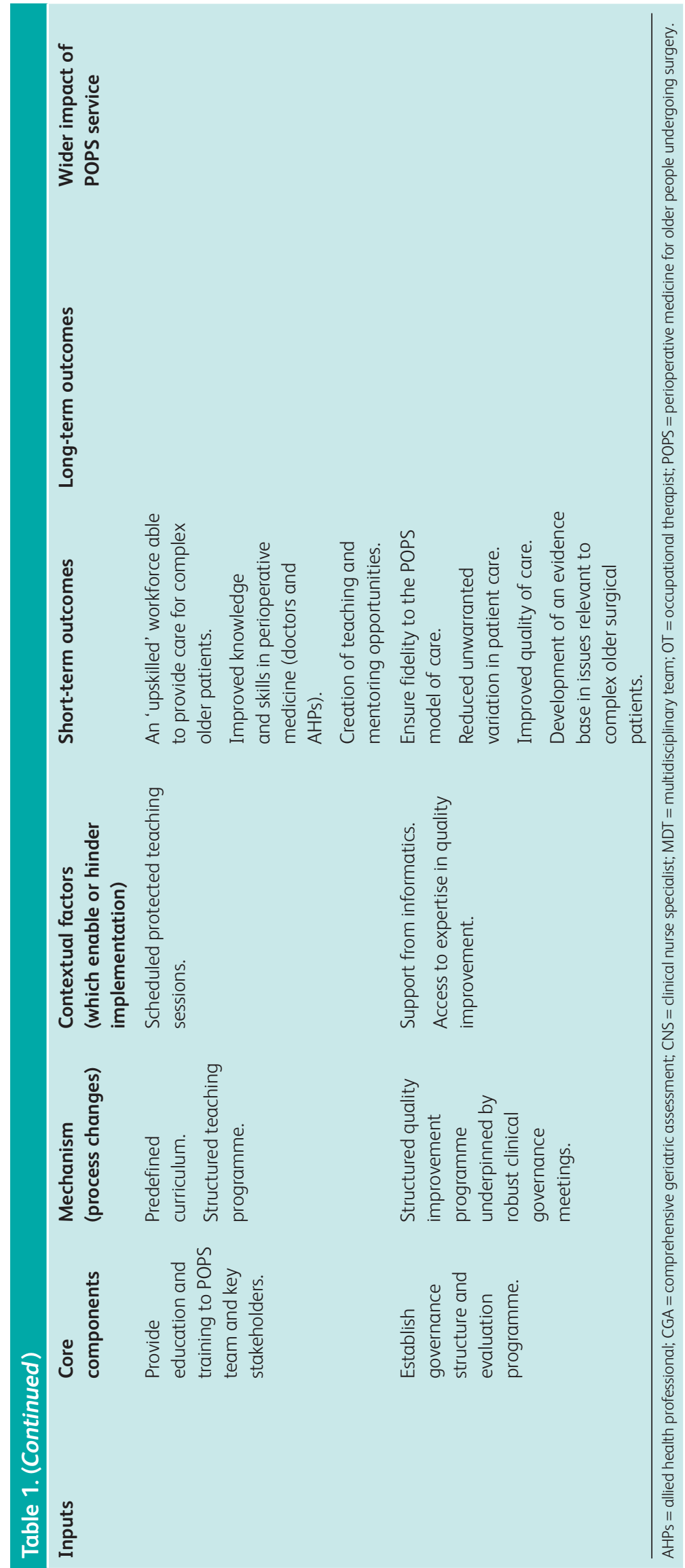


with other teams', was used to supplement a simple description of mechanism or activity 'joint board rounds'. This additional level of detail facilitated a sharing of vison as well as the tool or enabler to support implementation in clinical practice.

The second modification to the classic logic model structure, was the inclusion of contextual factors. Often absent from logic models, contextual factors are key to understanding how the delivery of a POPS intervention may differ between settings yet still achieve the intended clinical objectives. To address context, the panel described important barriers and/or enablers to equip users of the logic model with the necessary tools to overcome acknowledged barriers in POPS service development. Furthermore, inclusion of these contextual factors provided a framework for identification of poorly performing components of the service which can be used to inform quality improvement programmes. The fusion of logic model development with wider elements of implementation science, has facilitated the creation of a novel model style with the potential for more sustainable impact.

\section{Applying the logic model}

The logic model was used as a framework to translate POPS@ GSTFT to POPS@DGT, a district general hospital with notable differences in case mix, clinical pathways and workforce. The new service was implemented over a 12-month period (October 2017 to September 2018). The model was instrumental in ensuring the fidelity of the service to the original and pre-empting barriers through early identification of contextual factors. The overall success of the scale-up was reflected in an effective business case leading to initiation of POPS@DGT as a substantive service, funded by their surgical directorate (as of September 2018).

\section{Discussion}

This is the first study to our knowledge to report the process of developing and applying a logic model to support the scale-up of a perioperative service for older surgical patients. Through providing a structured approach to the translation of core components of a POPS service, taking context and mechanism into account and engaging a wide stakeholder group, this study facilitated the establishment of a new and substantively funded POPS service.

Logic models are well established in general service design and implementation literature, but are under-utilised in healthcare services where they are predominantly used to describe the establishment of new services. ${ }^{18,19}$ In comparison, there are fewer examples of the use of logic models to facilitate the wider scale-up of established services. Two such programmes are described in the literature; the World Health Organization / Centers for Disease Control and Prevention's vitamin and mineral interventions in public health project and the Ensemble, Prévenons I'Obésité Des Enfants' (EPODE) [Together let's Prevent Childhood Obesity] programme, the latter of which developed a model retrospectively after already spreading to 500 centres. ${ }^{20,21}$ Interestingly, both these studies formulated logic models featuring broad overarching performance indicators at a high level. In comparison, the POPS logic model described detailed activities and incorporated contextual factors to describe barriers and enablers to implementation providing a pragmatic approach to guide further implementation.

The study has limitations. The first limitation in the development of the POPS logic model relates to the stakeholder group. While the group was wide and representative of the perioperative pathway, a lack of resources prohibited the involvement of a national expert panel which may have offered further validation of the model. A second, related limitation was the use of just two different clinical settings (GSTFT and DGT). However, this preliminary work demonstrating feasibility and utility of the logic model in a single centre translation, now sets the scene for wider NHS scale-up. Third, longer-term follow-up of POPS@DGT is required to establish the longevity and sustainability of the newly implemented service facilitated through the logic model.

This study offers a practical, evidence-based model for translation of clinical innovation. The success that we observed in translating the POPS@GSTFT service toPOPS@DGT offers early evidence that this style of logic model is feasible and useful in designing service scale-up.

A logic model is the figurative 'black box' of a service and therefore acts as a platform for service evaluation and provides a framework for service scale-up. While POPS has been running in the parent site (GSTFT) for over a decade, its wider scale-up within the NHS has been slower than ideal. The development of the logic model facilitated an early step in this translation process. Refinement of the logic model through a national consensus study would be the next step in ensuring an adequate level of detail, validity and generalisability of the logic model. Alongside this iterative development of the model, clinical and implementation evaluation is required to assess both clinical effectiveness and sustainability of newly established POPS services.

\section{Conclusion}

This study shows the feasibility and utility of logic model development to inform translation of POPS services to a new setting. Further refinement and evaluation of the logic model is planned through engagement with a national network of POPS services to facilitate wider scale-up. While this paper describes the use of a logic model in the perioperative setting, it is anticipated that the logic model approach reported here could have utility in other clinical settings beyond perioperative services.

\section{Supplementary material}

Additional supplementary material may be found in the online version of this article at www.rcpjournals.org:

$$
\text { S1 - Panel members and affiliations. }
$$

\section{References}

1 Fowler A, Abbott T, Prowle J, Pearse R. Age of patients undergoing surgery. Br J Surg 2019;106:1012-8.

2 Joughin A, Partridge J, O'Halloran T, Dhesi J. Where are we now in perioperative medicine? Results from a repeated UK survey of geriatric medicine delivered services for older people. Age Ageing 2019;48:458-62.

3 Thillainadesan J, Hilmer S, Close ] et al. Geriatric medicine services for older surgical patients in acute hospitals: Results from a binational survey. Australas ] Ageing 2019:[Epub ahead of print].

4 Eamer $\mathrm{G}$, Taheri A, Chen $\mathrm{S}$ et al. Comprehensive geriatric assessment for older people admitted to a surgical service. Cochrane Database Syst Rev 2018;1:CD012485.

5 Braude P, Goodman A, Elias T et al. Evaluation and establishment of a ward-based geriatric liaison service for older urological surgical patients: POPS-Urology (Proactive Care of Older People Undergoing Surgery). BJU Int 2017;120:123-9. 
6 Partridge J, Harari D, Martin F et al. Randomised clinical trial of comprehensive geriatric assessment and optimisation in vascular surgery. BJS 2017;104:679-98.

7 Vilches-Moraga A, Fox J. Geriatricians and the older emergency general surgical patient: proactive assessment and patient centred interventions - Salford-POP-GS. Aging Clin Expl Res 2018;30:277-82.

8 Pearce L, Bunni J, McCarthy K, Hewitt J. Surgery in the older person: Training needs for the provision of multidisciplinary care. Ann R Coll Surg Engl 2016;98:367-70.

9 Royal College of Anaesthetists. Perioperative medicine: The pathway to better surgical care. London: RCoA, 2015. www.rcoa.ac.uk/sites/ default/files/PERIOP-2014.pdf [Accessed 10 March 2019].

10 Royal College of Physicians. Future Hospital: Caring for medical patients. London: RCP, 2013. www.rcplondon.ac.uk/projects/ outputs/future-hospital-commission [Accessed 10 March 2019].

11 Morris Z, Wooding S, Grant J. The answer is 17 years, what is the question: understanding time lags in translational research. JRSM Open 2011;104:510-20.

12 Horton T, Illingworth ], Warburton W. The spread challenge: How to support the successful uptake of innovations and improvements in health care. London: The Health Foundation, 2018. www.health. org.uk/publications/the-spread-challenge [Accessed 25 February 2019].

13 Albury D, Beresford T, Dew S et al. Against the odds: Successfully scaling innovation in the NHS. Innovation Unit Press, 2018. www. health.org.uk/publications/against-the-odds-successfully-scalinginnovation-in-the-nhs [Accessed 25 Feb 2019].

14 Stephens T, Peden C, Pearse R et al. Improving care at scale: process evaluation of a multi-component quality improvement intervention to reduce mortality after emergency abdominal surgery (EPOCH trial). Implement Sci 2018;13:142.

15 WK Kellogg Foundation. Logic model development guide. Battle Creek: WK Kellogg Foundation, 2004. www.wkkf.org/resourcedirectory/resource/2006/02/wk-kellogg-foundation-logic-modeldevelopment-guide [Accessed 22 February 2019].

16 Peyton D, Scicchitano M. Devil is in the details: Using logic models to investigate program process. Eval Program Plan 2017;65:156-62.

17 Moore G, Audrey S, Barker M et al. Process evaluation of complex interventions: Medical Research Council guidance. BMJ 2015;350:h1258.

18 Chanfreau-Coffinier C, Peredo ], Russell MM et al. A logic model for precision medicine implementation informed by stakeholder views and implementation science. Genet Med 2018;21:1139-54.

19 Abimola S, Li C, Mitchell M et al. On the same page: co-designing the logic model of a telehealth service for children in rural and remote Australia. Digital health 2019;5:1-7.

20 De-Regil L, Pena-Rosas J, Flores-Ayala R, Socorro Jefferds M. Development and use of the generic WHO/CDC logic model for vitamin and mineral interventions in public health programmes. Public Health Nutr 2014:17:634-9.

21 Van Koperen T, Jebb S, Summerbell C et al. Characterizing the EPODE logic model: unravelling the past and informing the future. Obes Rev 2012:14:162-70.

Address for correspondence: Dr Emily V Jasper, Guy's Hospital, Great Maze Pond, London SE1 9RT, UK.

Email: emily.jasper@gstt.nhs.uk

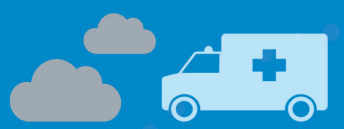

\section{Outpatients: the future - adding value through sustainability}

Published in 2018, this report from the Royal College of Physicians argues for

new models of outpatient care.

Download the report and

supplementary case studies at

www.rcplondon.ac.uk/outpatients-future-sustainability 